\title{
Determination of 105 pesticide residues by GC/MS/MS
}

\author{
W. S. Kim ${ }^{\star}$, J. A. Do' ${ }^{1}$ H. J. Lee, J. Y. Lee, S. J. Yang ${ }^{2}$ and S.H. Lee \\ Test \& Analytical Laboratory, Busan Regional Food \& Drug Administration, Busan 608-829, Korea \\ ${ }^{1}$ National institute of Food and Drug Safety Evaluation, Korea Food \& Drug Administration, Seoul, 122-704, Korea \\ ${ }^{2}$ Agilent Korea, Seoul, 135-280, Korea \\ ${ }^{3}$ Medical Device Safety Bureau, Korea Food \& Drug Administration, Seoul, 122-704, Korea \\ (Received September 7, 2009; Accepted June 10, 2010)
}

\section{GC/MS/MS를 이용한 105종의 잔류농약 분석에 관한 연구 \\ 김우성 ${ }^{\star}$ 도정아 ${ }^{1} \cdot$ 이희정 $\cdot$ 이주영 $\cdot$ 양승주 ${ }^{2} \cdot$ 이선희 $^{3}$ \\ 부산지방식품의약품안전청 시험분석센터, ${ }^{1}$ 식품의약품안전평가원 화학물질과 \\ ${ }^{2}$ 에질런트 코리아, ${ }^{3}$ 식품의약품안전청 의약품안전국 \\ (2009.9. 7. 접수, 2010. 6. 10. 승인)}

\begin{abstract}
A multi-residual method using gas chromatography coupled with mass spectrometry (GC/MS/ MS) was developed for the analysis of 105 pesticides. This method was tested on lemons, beans and other vegetables. The pretreatment of these pesticides was performed by liquid-liquid partition followed by cleanup with solid phase extraction cartridge (SPE Florisil), after acetonitrile extraction from matrices and sodium chloride $(15 \mathrm{~g}$ ) addition. The recovery ranged from $71.1 \%$ to $126.0 \%$ except for azinphosmethyl, famoxadone, fenamidone, flufenoxuron and triadimefon in lemons and from $72.5 \%$ to $124.5 \%$ in bean. In lemon, the limit of detection (LOD) and limit of quantification (LOQ) were $0.001 \sim 150 \mathrm{ng} /$ $\mathrm{mL}$ and $0.004 \sim 500 \mathrm{ng} / \mathrm{mL}$, respectively.

요 약: 레몬과 콩 그리고 채소류에서 105 개의 잔류농약을 다종 다성분 분석법으로 $\mathrm{GC} / \mathrm{MS} / \mathrm{MS}$ 분석하 였다. 각 시료로부터 acetonitrile로 1차 추출하고 $\mathrm{NaCl} 15 \mathrm{~g}$ 을 첨가한 다음 액-액 분배하였으며, Florisil 고체상(SPE) Cartridge로 정제하여 분석하였다. 레몬과 콩에서의 105개 농약의 회수율은 azinphos-methyl, famoxadone, fenamidone, flufenoxuron, triadimefon을 제외한 모든 농약에서 우수한 것으로 나타났는데 레 몬에서 71.1 126.0\%, 콩에서는 72.5 124.5\% 였다. 또한 레몬에서의 검출한계와 정량한계는 각각 $0.001 ~ 150$ $\mathrm{ng} / \mathrm{mL}$ 와 $0.004-500 \mathrm{ng} / \mathrm{mL}$ 으로 확인되었다.
\end{abstract}

Key words: multi-residual method, GC/MS/MS, limit of detection, limit of quantification

Corresponding author

Phone : +82-(0)51-610-6140 Fax : +82-(0)51-610-6159

E-mail : kwsh1964@kfda.go.kr 


\section{Introduction}

Agricultural chemicals synthesized to avoid damage due to blight and harmful insects are used to ensure the safety of agricultural products. However, with their beneficial characteristics, they also have adverse effects on the human body and environment. Public interest in this problem is high, as people are concerned about the reported effects of pesticides on

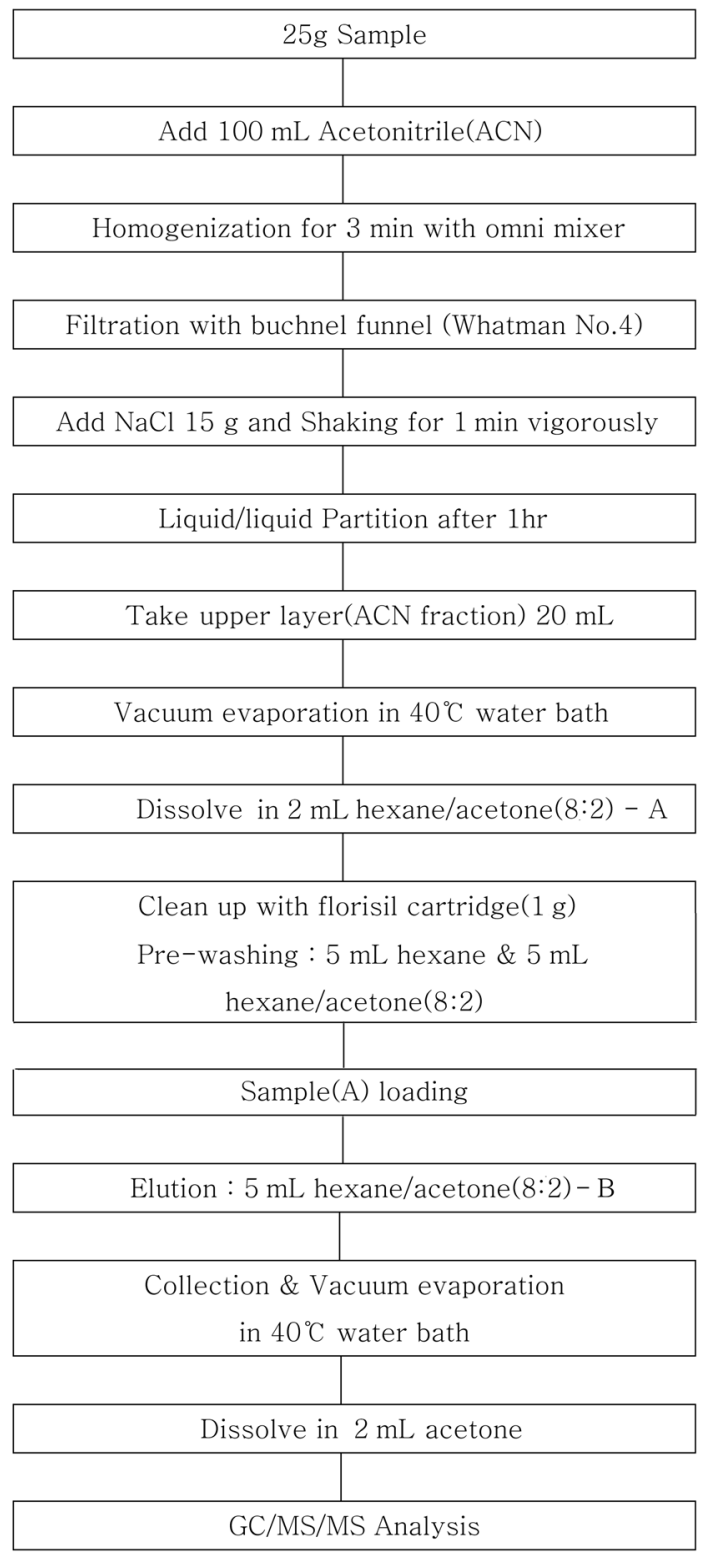

Fig. 1. Flow diagram of sample preparation procedure. their own bodies.

The pesticides that remain in most agricultural products decrease with time and through the pretreatment process; however, safety problems may occur due to the pesticides remaining in fruits and vegetables. Many procedures for extraction of these pesticides require extensive sample preparation, multiple extractions, extract purification and concentration before chromatographic analysis. ${ }^{1-4}$ In addition, various instruments are used to determine whether the trace amounts of pesticides in foodstuffs comply with the level required by legislation regulation. In recent years, some applications using GC, HPLC and mass selective detectors (GC/MS) have been described as rapid and reliable multi-residual methods for deter-mining pesticide levels. ${ }^{5-7}$ Mass spectrometry (MS) has become very popular in laboratories for detecting pesticides. Unfortunately, a full scan often does not provide enough sensitivity in real samples, but SIM (Selected Ion Monitoring), which improves sensitivity, considerably reduces the qualitative information, thus increasing the risk of false positives. ${ }^{8}$ The combination of GC with tandem mass spectrometry (GC/MS/MS) has been shown to be applicable to the analysis of trace amounts of contaminants like pesticides or metabolites in complex samples such as biological fluids, ${ }^{9-11}$ water ${ }^{12-13}$ or fruits and vegetables. ${ }^{14-15}$ This paper proposes a new method to determine the levels of 105 pesticides in beans and lemon. It is based on fast and simple acetonitrile extraction of the samples with SPE florisil clean up (Fig. 1) and GC/MS/MS analysis.

\section{Experimental}

\subsection{Reagents and standards}

Pesticide standards of analytical grade were purchased from Dr. Ehrenstorfer GmBH (Augsberg, Germany), Chem Service (West Chester, USA), Supelco (Bellefonte, USA), Riedel de Haen (Schnelldorf, Germany) or Wako (Japan). Individual stock solutions were prepared by dissolving $5 \sim 10 \mathrm{mg}$ of each compound in $10 \mathrm{~mL}$ acetone. A mixed solution with approximate concentration of $10 \mu \mathrm{g} / \mathrm{mL}$ in 
acetone was also prepared. Working standard solutions were prepared by diluting this mixed solution. Residue analysis grade acetonitrile and anhydrous sodium sulfate were purchased from Merck (Damstadt, Germany). Additionally, sodium chloride (Junsei) and Whatman filter paper, bond elute (MEGA BE-FL $1 \mathrm{~g} / 6 \mathrm{~mL}$, Varian) were used for cleanup.

\subsection{Instrumental analysis}

A Varian (Walnut Creek, CA, USA) CP-3800 gas chromatograph equipped with a triple Quadrupole mass spectrometer was used. Splitless injection was performed with purge delay time $1.5 \mathrm{~min}$ (Purge flow $100 \mathrm{~mL} / \mathrm{min}$ ). A Varian (5\% phenyl-95\% methyl silicone $)$ fused-silica column $(30 \mathrm{~m} \times 0.25 \mathrm{~mm} \times 0.25$ $\mu \mathrm{m}$ film thickness) was used. Other GC/MS/MS analysis conditions are as follows; helium carrier gas flow, $0.8 \mathrm{~mL} / \mathrm{min}$; injector temperature, $250{ }^{\circ} \mathrm{C}$; injection volume, $1 \mu \mathrm{L}$; ion source temperature, 200 ${ }^{\circ} \mathrm{C}$; solvent delay time, $4 \mathrm{~min}$; oven temperature condition: $70{ }^{\circ} \mathrm{C}$ for $3 \mathrm{~min}$, then $20^{\circ} \mathrm{C} / \mathrm{min}$ to $180{ }^{\circ} \mathrm{C}$ (holding for $8.5 \mathrm{~min}$ ) and $5{ }^{\circ} \mathrm{C} / \mathrm{min}$ to $300{ }^{\circ} \mathrm{C}$, held for $7.5 \mathrm{~min}$. The total run time was $40 \mathrm{~min}$. The common parameters for source, manifold and transfer line temperatures were $200{ }^{\circ} \mathrm{C}, 40{ }^{\circ} \mathrm{C}$ and $250^{\circ} \mathrm{C}$, respectively. The optimization of collisioninduced dissociation (CID) parameters was carried out in order to generate MS/MS spectra with a

Table 1. Instrumental analysis conditions of the 105 pesticides

\begin{tabular}{|c|c|c|c|c|c|c|c|c|c|}
\hline No. & Seg & Pesticides & $\begin{array}{l}\text { Retention } \\
\text { time }\end{array}$ & M.W. & $\begin{array}{l}\text { Precursor } \\
\text { ion }\end{array}$ & MRM1 & MRM2 & MRM3 & $\begin{array}{c}\text { Collision } \\
\text { energy }(\mathrm{eV})\end{array}$ \\
\hline 1 & 1 & Propoxur & 7.181 & 209 & 110 & 64 & 92 & & 15 \\
\hline 2 & 2 & Dichlobenil & 8.577 & 171 & 171 & 100 & 136 & & 20 \\
\hline 3 & 2 & Hexaflumuron & 9.013 & 461 & 202 & $110(30)$ & $174(15)$ & $146(20)$ & \\
\hline 4 & 2 & Lufenuron & 9.010 & 511 & 203 & $140(20)$ & $111(30)$ & $175(15)$ & \\
\hline 5 & 2 & Mevinphos & 9.092 & 224 & 192 & 127 & 164 & & 10 \\
\hline 6 & 2 & Nitrapyrin & 9.401 & 229 & 194 & 133 & 158 & & 20 \\
\hline 7 & 3 & Tecnazene & 10.742 & 259 & 203 & 85 & 143 & & 25 \\
\hline 8 & 4 & BHC-alpha & 11.965 & 288 & 181 & 145 & 109 & & 25 \\
\hline 9 & 4 & Chlorpropham & 11.301 & 213 & 213 & 171 & 127 & & 5 \\
\hline 10 & 4 & Dicloran & 12.241 & 206 & 206 & 148 & 176 & & 20 \\
\hline 11 & 4 & Diphenylamine & 11.076 & 169 & 169 & 169 & 167 & & 10 \\
\hline 12 & 4 & Ethalfluralin & 11.108 & 333 & 276 & 248 & 202 & & 10 \\
\hline 13 & 4 & Ethoprophos & 11.073 & 242 & 158 & 114 & 97 & & 20 \\
\hline 14 & 4 & Simazine & 12.282 & 201 & 201 & 173 & 186 & & 10 \\
\hline 15 & 4 & Thiometon & 12.051 & 246 & 125 & 79 & 93 & & 20 \\
\hline 16 & 4 & Trifluralin & 11.245 & 335 & 306 & 206 & 264 & & 20 \\
\hline 17 & 5 & BHC-beta & 12.537 & 288 & 181 & 109 & 145 & & 30 \\
\hline 18 & 5 & BHC-delta & 13.434 & 288 & 181 & 145 & 109 & & 10 \\
\hline 19 & 5 & BHC-gamma & 12.740 & 288 & 181 & 145 & 109 & & 10 \\
\hline 20 & 5 & Diazinon & 12.772 & 304 & 304 & 179 & 137 & & 30 \\
\hline 21 & 5 & Etrimfos & 13.220 & 292 & 181 & 153 & 98 & & 5 \\
\hline 22 & 5 & Flufenoxuron(2) & 13.424 & 489 & 126 & $98(15)$ & $106(10)$ & & \\
\hline 23 & 5 & Fonofos & 12.873 & 246 & 246 & $109(20)$ & $137(10)$ & & \\
\hline 24 & 5 & Isazofos & 13.126 & 313 & 161 & 119 & 146 & & 10 \\
\hline 25 & 5 & Quintozene & 12.622 & 293 & 237 & 143 & 119 & & 20 \\
\hline 26 & 5 & Tebupirimfos & 13.384 & 318 & 261 & 137 & 153 & & 15 \\
\hline 27 & 5 & Tefluthrin & 13.067 & 419 & 177 & 127 & 157 & & 5 \\
\hline 28 & 6 & Acetochlor & 14.060 & 269 & 223 & 132 & 147 & & 15 \\
\hline 29 & 6 & Alachlor & 14.317 & 269 & 188 & 131 & 160 & & 15 \\
\hline 30 & 6 & Chlorpyrifos-methyl & 14.136 & 321 & 286 & 93 & 271 & & 20 \\
\hline
\end{tabular}


Table 1. Continued

\begin{tabular}{|c|c|c|c|c|c|c|c|c|c|}
\hline No. & Seg & Pesticides & $\begin{array}{l}\text { Retention } \\
\text { time }\end{array}$ & M.W. & $\begin{array}{l}\text { Precursor } \\
\text { ion }\end{array}$ & MRM1 & MRM2 & MRM3 & $\begin{array}{l}\text { Collision } \\
\text { energy }(\mathrm{eV})\end{array}$ \\
\hline 31 & 6 & Dimethenamid & 13.990 & 276 & 230 & 154 & 137 & & 20 \\
\hline 32 & 6 & Dithiopyr & 14.570 & 401 & 286 & 210 & 238 & & 15 \\
\hline 33 & 6 & Formothion & 13.742 & 257 & 170 & $93(15)$ & $125>79(10)$ & & \\
\hline 34 & 6 & Heptachlor & 14.615 & 370 & 272 & 237 & 239 & & 20 \\
\hline 35 & 6 & Metalaxyl & 14.502 & 279 & 206 & 132 & 162 & & 10 \\
\hline 36 & 6 & Parathion-methyl & 14.326 & 263 & 263 & $109(10)$ & $127(20)$ & $246(5)$ & \\
\hline 37 & 6 & Propanil & 14.034 & 217 & 161 & 99 & 126 & & 30 \\
\hline 38 & 6 & Propisochlor & 14.435 & 284 & 162 & 119 & 144 & & 20 \\
\hline 39 & 6 & Tolclofos-methyl & 14.340 & 300 & 265 & 250 & 93 & & 20 \\
\hline 40 & 6 & Vinclozolin & 14.215 & 285 & 285 & 213 & 186 & & 10 \\
\hline 41 & 7 & Chlorpyrifos & 15.434 & 349 & 314 & 258 & 285 & & 15 \\
\hline 42 & 7 & Dichlofluanid & 15.236 & 332 & 224 & 123 & 77 & & 25 \\
\hline 43 & 7 & Diethofencarb & 15.426 & 267 & 225 & 196 & 168 & & 5 \\
\hline 44 & 7 & Dimethylvinphos & 15.513 & 332 & 295 & 109 & 280 & & 20 \\
\hline 45 & 7 & Esprocarb & 15.232 & 265 & 222 & 162 & 151 & & 5 \\
\hline 46 & 7 & Fenitrothion : MEP & 14.997 & 277 & 277 & 260 & 109 & & 10 \\
\hline 47 & 7 & Fosthiazate(2) & 16.187 & 283 & 195 & 103 & 139 & & 10 \\
\hline 48 & 7 & Malathion & 15.195 & 330 & 173 & 99 & 127 & & 10 \\
\hline 49 & 7 & Methyl pentachlorophenyl sulfide & 15.241 & 296 & 296 & 246 & 263 & & 30 \\
\hline 50 & 7 & Metolachlor & 15.407 & 283 & 162 & 133 & 134 & & 10 \\
\hline 51 & 7 & Pirimiphos-ethyl & 16.014 & 333 & 333 & 180 & 163 & & 10 \\
\hline 52 & 7 & Pirimiphos-methyl & 14.864 & 305 & 305 & 180 & 233 & & 15 \\
\hline 53 & 7 & Tetraconazole & 15.721 & 372 & 336 & 218 & 191 & & 15 \\
\hline 54 & 7 & Thiazopyr & 15.239 & 396 & 128 & $128(5)$ & $85(25)$ & & \\
\hline 55 & 7 & Triadimefon & 15.765 & 293 & 208 & $111(30)$ & 181(15) & & \\
\hline 56 & 8 & Chlorfenvinphos & 16.744 & 358 & 267 & 159 & 203 & & 15 \\
\hline 57 & 8 & Heptachlor-epoxide & 16.818 & 386 & 353 & 263 & 282 & & 15 \\
\hline 58 & 8 & Isofenphos & 16.667 & 345 & 213 & 121 & 185 & & 10 \\
\hline 59 & 8 & Mecarbam & 16.811 & 329 & 131 & 74 & 86 & & 15 \\
\hline 60 & 8 & Penconazole & 16.659 & 283 & 248 & $157(30)$ & 248 & & 5 \\
\hline 61 & 8 & Phenthoate : PAP & 16.920 & 320 & 274 & 121 & 246 & & 10 \\
\hline 62 & 8 & Procymidone & 17.088 & 283 & 283 & 96 & 283 & & 10 \\
\hline 63 & 8 & Tolyfluanid & 16.754 & 346 & 238 & 91 & 137 & & 35 \\
\hline 64 & 9 & $\mathrm{o}, \mathrm{p}-\mathrm{DDE}$ & 17.593 & 316 & 246 & 176 & 246 & & 30 \\
\hline 65 & 9 & Paclobutrazol & 17.681 & 293 & 236 & 125 & 167 & & 10 \\
\hline 66 & 10 & Carboxin & 18.855 & 235 & 143 & 114 & 143 & 87 & 10 \\
\hline 67 & 10 & Chinomethionat & 18.797 & 234 & 206 & 148 & 121 & & 25 \\
\hline 68 & 10 & Dieldrin & 18.880 & 378 & 263 & 193(30) & $228(20)$ & & \\
\hline 69 & 10 & Endosulfan-alpha & 17.986 & 404 & 195 & 125 & 159 & & 25 \\
\hline 70 & 10 & Myclobutanil & 18.749 & 288 & 179 & 125 & 152 & & 10 \\
\hline 71 & 10 & Napropamide & 18.149 & 271 & 271 & 72 & 128 & & 10 \\
\hline 72 & 10 & $\mathrm{o}, \mathrm{p}-\mathrm{DDD}$ & 18.883 & 318 & 235 & 199 & 165 & & 20 \\
\hline 73 & 10 & $\mathrm{p}, \mathrm{p}-\mathrm{DDE}$ & 18.634 & 316 & 246 & 176 & 211 & & 25 \\
\hline 74 & 10 & Prothiofos & 18.323 & 344 & 267 & 221 & 239 & & 20 \\
\hline 75 & 10 & Thifluzamide & 18.597 & 528 & 194 & 125 & 166 & & 20 \\
\hline 76 & 11 & Chlorobenzilate & 19.698 & 324 & 251 & 111 & 139 & & 30 \\
\hline 77 & 11 & Endosulfan-beta & 19.919 & 404 & 241 & 170 & 206 & & 20 \\
\hline
\end{tabular}


Table 1. Continued

\begin{tabular}{|c|c|c|c|c|c|c|c|c|c|}
\hline No. & Seg & Pesticides & $\begin{array}{l}\text { Retention } \\
\text { time }\end{array}$ & M.W. & $\begin{array}{l}\text { Precursor } \\
\text { ion }\end{array}$ & MRM1 & MRM2 & MRM3 & $\begin{array}{c}\text { Collision } \\
\text { energy }(\mathrm{eV})\end{array}$ \\
\hline 78 & 11 & Endrin & 19.576 & 378 & 263 & 193 & 228 & & 30 \\
\hline 79 & 11 & Fenoxanil & 19.370 & 329 & 189 & 125 & 154 & & 15 \\
\hline 80 & 11 & Fluacrypyrim & 20.186 & 426 & 145 & 115 & 102 & & 15 \\
\hline 81 & 12 & Carbophenothion & 20.893 & 342 & 342 & $157(10)$ & $296(5)$ & & \\
\hline 82 & 12 & Edifenphos & 21.012 & 310 & 173 & 109 & 155 & & 10 \\
\hline 83 & 12 & Norflurazon & 20.965 & 303 & 145 & 95 & 125 & & 15 \\
\hline 84 & 12 & p,p-DDT & 21.295 & 352 & 235 & 165 & 199 & & 20 \\
\hline 85 & 12 & Triazophos & 20.509 & 313 & 161 & 106 & 92 & & 15 \\
\hline 86 & 13 & Azinphos-methyl & 24.292 & 317 & 160 & 132 & 104 & & 5 \\
\hline 87 & 13 & Bifenthrin & 22.868 & 422 & 181 & 166 & 167 & & 15 \\
\hline 88 & 13 & Bromopropylate & 22.985 & 426 & 341 & 183 & 185 & & 10 \\
\hline 89 & 13 & EPN & 22.967 & 323 & 157 & 77 & 110 & & 15 \\
\hline 90 & 13 & Etoxazole & 23.179 & 359 & 141 & $112(20)$ & $113(15)$ & $141(20)$ & \\
\hline 91 & 13 & Fenamidone & 23.326 & 311 & 238 & 103 & 238 & & 30 \\
\hline 92 & 13 & Fenoxycarb & 23.083 & 301 & 186 & 109 & 157 & & 15 \\
\hline 93 & 13 & Fenpropathrin & 23.235 & 349 & 265 & $210(10)$ & $181(20)$ & & \\
\hline 94 & 13 & Indanofan & 23.482 & 341 & 139 & 75 & 111 & & 30 \\
\hline 95 & 13 & Phosalone & 24.111 & 367 & 182 & $111(25)$ & $138(10)$ & $102(25)$ & \\
\hline 96 & 13 & Phosmet (PMP) : Imidan & 22.891 & 317 & 160 & 132 & 104 & & 15 \\
\hline 97 & 13 & Tetradifon & 23.939 & 354 & 356 & 159 & 229 & & 20 \\
\hline 98 & 14 & Fenarimol & 25.149 & 330 & 251 & 139 & 111 & & 25 \\
\hline 99 & 14 & Fluquinconazole & 26.571 & 376 & 340 & 286 & 298 & & 25 \\
\hline 100 & 14 & Pyrazophos & 25.085 & 373 & 221 & 149 & 193 & & 15 \\
\hline 101 & 15 & Cypermethrin(3) & 27.964 & 415 & 181 & 152 & 127 & & 30 \\
\hline 102 & 15 & Famoxadone & 31.782 & 374 & 196 & 167 & 153 & & 15 \\
\hline 103 & 15 & Flucythrinate & 28.242 & 451 & 199 & 107 & 157 & & 15 \\
\hline 104 & 15 & Halfenprox & 28.030 & 477 & 265 & $265(10)$ & $131(25)$ & $237(15)$ & \\
\hline 105 & 15 & Indoxacarb & 30.821 & 528 & 150 & 123 & 114 & & 25 \\
\hline
\end{tabular}

relative abundance of the parent ion, and CID gas pressure was $1.5 \mathrm{~m}$ Torr. In EI mode (Ionization energy : $70 \mathrm{eV}$ ), the filament current was set to 50 $\mu \mathrm{A}$. The MRM parameters are summarized in Table 1. ${ }^{14}$ MRM 1 was used for quantification, and the intensity ratio of MRM 1, MRM 2 and MRM 3 was used as the confirmatory parameter.

\subsection{Sample preparation}

The extraction method was proposed by KFDA (Food Code No. 83). ${ }^{15}$ It is summarized in Fig. 1. The sample was dissolved in $2 \mathrm{~mL}$ (A) of hexane/ acetone (8:2) and loaded on Bond elute (florisil, $1 \mathrm{~g} /$ $6 \mathrm{~mL}$ ), and then eluted with hexane/acetone (8:2) 5 $\mathrm{mL}$ (B), which was previously conditioned with 5
$\mathrm{mL}$ of hexane, and $5 \mathrm{~mL}$ of hexane/acetone (8:2). 7 $\mathrm{mL}$ of hexane/acetone $(8: 2)(\mathrm{A}+\mathrm{B}=\mathrm{C})$ were combined. $\mathrm{C}$ solutions were dried on a rotary evaporator. An Omni Macro Homogenizer with high-speed was used.

\section{Results and Discussion}

\subsection{Optimization of $\mathrm{MS} / \mathrm{MS}$ experimental} conditions

The GC/MS/MS measurement method is usually composed of some groups of MRM ions by the system software from Varian instrumental equipment (GC/MS/MS). That is time segment of start and end times of each function can be defined as retention 


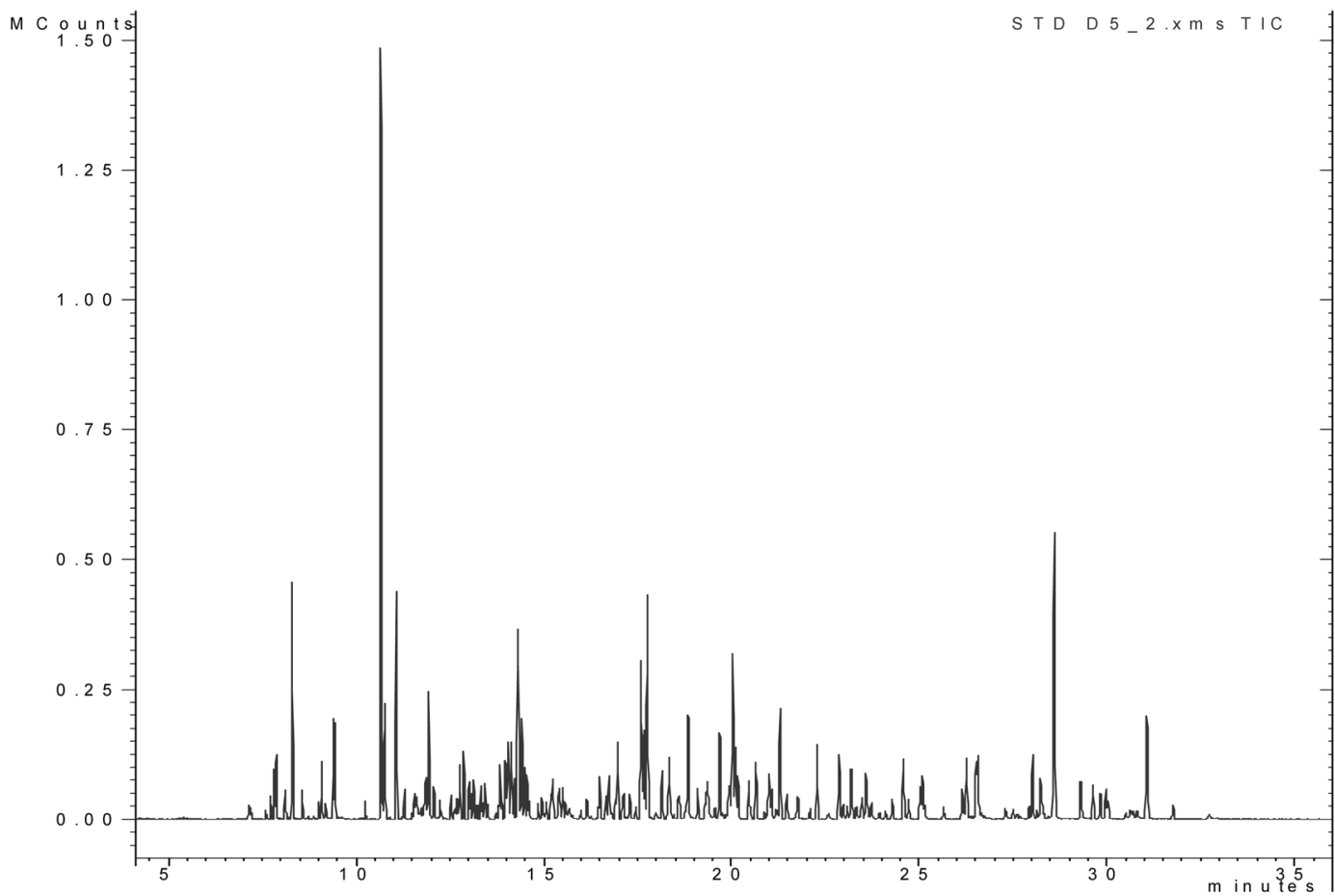

Fig. 2. Typical chromatogram of a standard mixture solution including 105 pesticides.

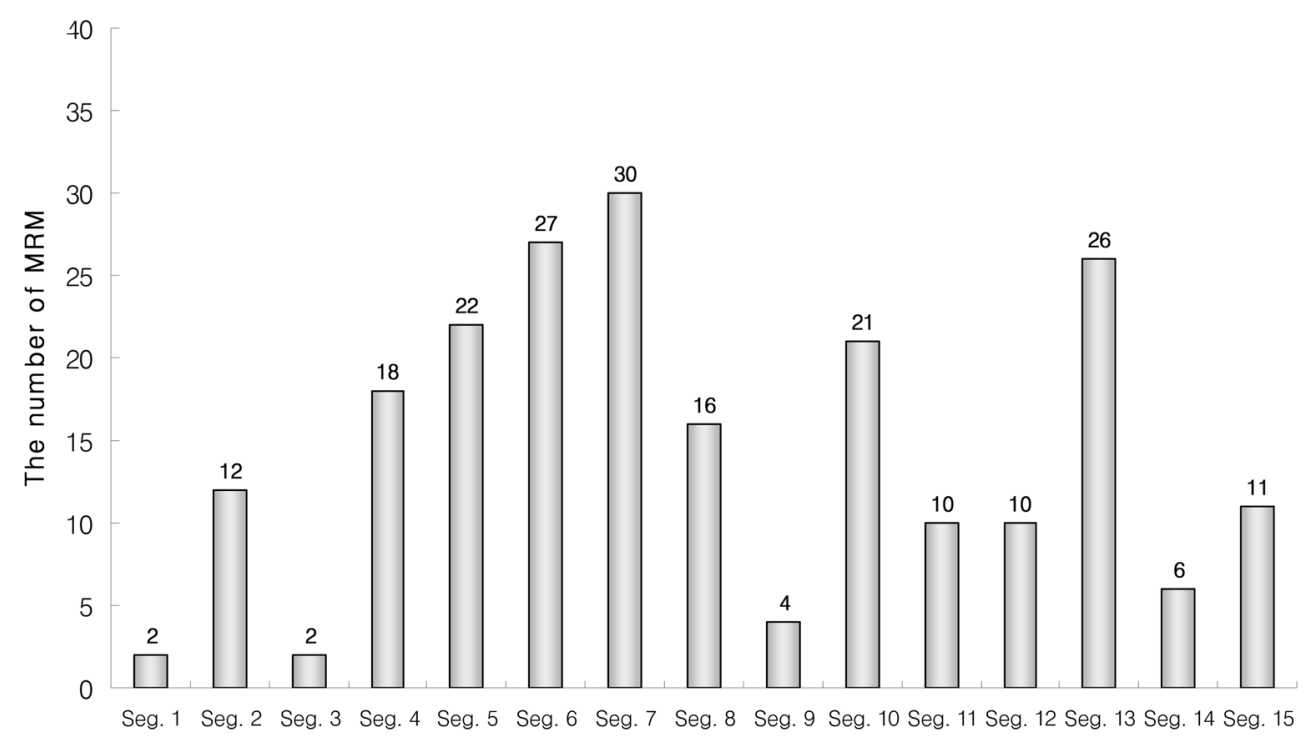

Fig. 3. MRM distribution of the 15 acquisition groups.

times of individual peaks. A measurement method can be constructed with 1 to 32 functions that can allow 40 transition ions to be monitored simultaneously (same segment time) without significant loss of sensitivity (although the maximum number of transition ions is 200). In practical terms, the chromatographic peak shape, which depends on scan speed, restricts the number of simultaneous analyses. Following conditions from a previous paper (Fernandez Moreno et al. 2008), we established a rapid and 
Table 2. Recovery and validation results of 105 pesticides in lemon and bean $(n=3)$

\begin{tabular}{|c|c|c|c|c|c|c|c|c|c|c|}
\hline \multirow[b]{2}{*}{ No. } & \multirow[b]{2}{*}{ Pesticide } & \multirow[b]{2}{*}{$\begin{array}{l}\text { Treatment } \\
\text { conc.(ppm) }\end{array}$} & \multicolumn{2}{|c|}{ Bean } & \multicolumn{2}{|c|}{ Lemon } & \multicolumn{2}{|c|}{ Bean } & \multicolumn{2}{|c|}{ Lemon } \\
\hline & & & $\begin{array}{c}\text { Recovery } \\
(\%)\end{array}$ & $\begin{array}{l}\mathrm{RSD} \\
(\%)\end{array}$ & $\begin{array}{c}\text { Recovery } \\
(\%)\end{array}$ & $\begin{array}{l}\text { RSD } \\
(\%)\end{array}$ & $\mathrm{ng} / \mathrm{g}$ & $\mathrm{ng} / \mathrm{g}$ & $\mathrm{ng} / \mathrm{g}$ & $\mathrm{ng} / \mathrm{g}$ \\
\hline 1 & Acetochlor & 1.20 & 88.6 & 4.1 & 108.7 & 3.0 & 0.007 & 0.023 & 0.005 & 0.017 \\
\hline 2 & Alachlor & 0.53 & 89.1 & 4.6 & 108.8 & 2.3 & 0.012 & 0.039 & 0.456 & 1.521 \\
\hline 3 & Azinphos-methyl & 0.53 & 77.9 & 6.2 & 67.5 & 6.3 & 4.660 & 15.534 & 9.430 & 31.434 \\
\hline 4 & BHC-alpha & 0.27 & 86.8 & 2.4 & 103.8 & 4.8 & 0.003 & 0.010 & 0.002 & 0.008 \\
\hline 5 & BHC-beta & 0.40 & 83.5 & 3.1 & 109.0 & 3.9 & 0.010 & 0.034 & 0.480 & 1.599 \\
\hline 6 & BHC-delta & 0.40 & 78.2 & 3.8 & 102.2 & 4.5 & 0.013 & 0.043 & 0.616 & 2.055 \\
\hline 7 & BHC-gamma & 0.27 & 82.2 & 3.5 & 101.8 & 2.3 & 0.010 & 0.035 & 0.600 & 2.002 \\
\hline 8 & Bifenthrin & 0.13 & 85.0 & 6.2 & 99.0 & 1.5 & 0.158 & 0.525 & 0.213 & 0.711 \\
\hline 9 & Bromopropylate & 0.27 & 112.5 & 6.9 & 129.0 & 2.7 & 0.005 & 0.016 & 0.004 & 0.012 \\
\hline 10 & Carbophenothion & 0.40 & 98.5 & 3.0 & 117.7 & 1.8 & 0.012 & 0.039 & 0.008 & 0.027 \\
\hline 11 & Carboxin & 0.13 & 72.5 & 2.3 & 85.0 & 1.7 & 15.385 & 51.282 & 7.792 & 25.974 \\
\hline 12 & Chionomethionat & 0.67 & 79.7 & 3.7 & 72.8 & 1.4 & 0.087 & 0.288 & 10.830 & 36.101 \\
\hline 13 & Chlorfenvinphos & 0.53 & 93.5 & 4.3 & 106.6 & 1.8 & 0.006 & 0.019 & 0.005 & 0.015 \\
\hline 14 & Chlorobenzilate & 0.40 & 108.8 & 5.1 & 127.7 & 2.0 & 0.001 & 0.005 & 0.001 & 0.004 \\
\hline 15 & Chlorphopham & 0.80 & 90.8 & 6.5 & 119.6 & 4.0 & 0.054 & 0.180 & 0.028 & 0.093 \\
\hline 16 & Chlorpyrifos & 0.40 & 89.5 & 3.5 & 113.5 & 2.2 & 0.005 & 0.016 & 0.003 & 0.011 \\
\hline 17 & Chlorpyrifos-methyl & 1.33 & 86.1 & 4.4 & 113.7 & 2.1 & 0.004 & 0.013 & 0.148 & 0.494 \\
\hline 18 & Cypermethrin & 1.07 & 106.9 & 3.9 & 124.9 & 0.5 & 7.565 & 25.217 & 4.272 & 14.241 \\
\hline 19 & Diazinon & 2.00 & 92.4 & 2.3 & 109.6 & 2.5 & 0.009 & 0.031 & 0.007 & 0.023 \\
\hline 20 & Dichlobenil & 0.13 & 81.0 & 2.6 & 101.5 & 2.3 & 0.001 & 0.002 & 0.001 & 0.003 \\
\hline 21 & Dichlolofluanid & 0.53 & 84.9 & 6.6 & 104.5 & 5.1 & 0.007 & 0.024 & 0.016 & 0.053 \\
\hline 22 & Dichloran & 0.67 & 100.6 & 6.4 & 128.4 & 4.0 & 0.014 & 0.046 & 0.013 & 0.043 \\
\hline 23 & Dieldrin & 0.67 & 79.8 & 0.0 & 96.7 & 1.2 & 0.026 & 0.085 & 0.019 & 0.064 \\
\hline 24 & Diethofencarb & 0.40 & 88.8 & 4.0 & 93.2 & 3.3 & 0.249 & 0.829 & 0.187 & 0.623 \\
\hline 25 & Dimethenamid & 2.00 & 89.1 & 3.9 & 105.3 & 2.4 & 0.005 & 0.015 & 0.004 & 0.012 \\
\hline 26 & Dimethylvinphos & 0.67 & 86.9 & 5.0 & 94.4 & 3.2 & 0.004 & 0.012 & 0.003 & 0.012 \\
\hline 27 & Diphenylamine & 0.13 & 97.5 & 4.0 & 110.5 & 2.3 & 0.025 & 0.084 & 0.037 & 0.123 \\
\hline 28 & Dithiopyr & 2.00 & 85.9 & 3.0 & 104.4 & 3.1 & 0.007 & 0.024 & 0.006 & 0.019 \\
\hline 29 & Edifenphos & 0.27 & 102.3 & 3.0 & 111.0 & 1.3 & 0.003 & 0.009 & 0.003 & 0.011 \\
\hline 30 & Endosulfan-alpha & 0.67 & 82.3 & 2.2 & 95.4 & 3.9 & 0.059 & 0.197 & 0.044 & 0.147 \\
\hline 31 & Endosulfan-beta & 0.67 & 82.2 & 2.9 & 93.5 & 3.0 & 0.086 & 0.285 & 0.057 & 0.191 \\
\hline 32 & Endrin & 0.67 & 92.3 & 3.3 & 108.1 & 2.0 & 0.020 & 0.068 & 0.015 & 0.050 \\
\hline 33 & EPN & 0.27 & 93.5 & 6.4 & 111.3 & 1.6 & 0.030 & 0.100 & 0.773 & 2.576 \\
\hline 34 & Esprocarb & 0.53 & 85.1 & 2.9 & 101.0 & 4.8 & 0.182 & 0.606 & 0.075 & 0.249 \\
\hline 35 & Ethalfuralin & 0.40 & 86.5 & 6.5 & 116.7 & 2.0 & 0.019 & 0.064 & 0.010 & 0.034 \\
\hline 36 & Ethoprophos & 0.27 & 96.5 & 5.0 & 88.5 & 0.8 & 0.288 & 0.962 & 0.959 & 3.197 \\
\hline 37 & Etoxazole & 0.27 & 104.3 & 3.8 & 115.3 & 1.6 & 200.000 & 666.667 & 4.196 & 13.986 \\
\hline 38 & Etrimfos & 1.33 & 87.4 & 6.7 & 113.2 & 2.5 & 0.101 & 0.337 & 0.060 & 0.201 \\
\hline 39 & Famoxadone & 1.47 & 88.6 & 4.5 & 49.9 & 1.2 & 1.974 & 6.580 & 2.510 & 8.368 \\
\hline 40 & Fenamidone & 0.67 & 122.0 & 3.3 & 41.1 & 0.3 & 0.013 & 0.043 & 0.013 & 0.045 \\
\hline 41 & Fenarimol & 0.40 & 100.0 & 3.5 & 96.7 & 2.8 & 0.010 & 0.032 & 0.012 & 0.041 \\
\hline 42 & Fenitrothion & 0.27 & 85.5 & 4.5 & 109.8 & 3.5 & 0.015 & 0.051 & 0.011 & 0.035 \\
\hline 43 & Fenoxanil & 0.40 & 91.2 & 4.0 & 100.7 & 2.6 & 0.035 & 0.115 & 0.049 & 0.165 \\
\hline 44 & Fenoxycarb & 1.47 & 105.8 & 3.0 & 113.3 & 2.6 & 0.071 & 0.237 & 3.766 & 12.553 \\
\hline 45 & Fenpropathrin & 0.80 & 86.4 & 3.3 & 115.1 & 0.9 & 0.025 & 0.085 & 0.019 & 0.063 \\
\hline 46 & Fluacrypyrim & 0.53 & 89.6 & 4.5 & 104.9 & 1.3 & 1.443 & 4.811 & 1.612 & 5.373 \\
\hline
\end{tabular}


Table 2. Continued

\begin{tabular}{|c|c|c|c|c|c|c|c|c|c|c|}
\hline \multirow[b]{2}{*}{ No. } & \multirow[b]{2}{*}{ Pesticide } & \multirow[b]{2}{*}{$\begin{array}{l}\text { Treatment } \\
\text { conc.(ppm) }\end{array}$} & \multicolumn{2}{|c|}{ Bean } & \multicolumn{2}{|c|}{ Lemon } & \multicolumn{2}{|c|}{ Bean } & \multicolumn{2}{|c|}{ Lemon } \\
\hline & & & $\begin{array}{c}\text { Recovery } \\
(\%)\end{array}$ & $\begin{array}{c}\text { RSD } \\
(\%)\end{array}$ & $\begin{array}{c}\text { Recovery } \\
(\%)\end{array}$ & $\begin{array}{c}\text { RSD } \\
(\%)\end{array}$ & $\mathrm{ng} / \mathrm{g}$ & $\mathrm{ng} / \mathrm{g}$ & $\mathrm{ng} / \mathrm{g}$ & $\mathrm{ng} / \mathrm{g}$ \\
\hline 47 & Flucythrinate & 0.53 & 107.3 & 5.7 & 115.8 & 0.8 & 0.017 & 0.058 & 0.369 & 1.231 \\
\hline 48 & Flufenoxuron & 2.67 & 92.5 & 3.1 & 66.5 & 1.5 & 0.015 & 0.049 & 0.024 & 0.081 \\
\hline 49 & Fluquinconazole & 0.53 & 109.6 & 4.3 & 93.0 & 4.1 & 0.003 & 0.010 & 0.004 & 0.013 \\
\hline 50 & Fonofos & 0.67 & 92.0 & 4.3 & 116.9 & 2.3 & 0.002 & 0.006 & 0.001 & 0.004 \\
\hline 51 & Formothion & 0.27 & 85.5 & 4.6 & 88.5 & 1.5 & 1.599 & 5.330 & 1.548 & 5.161 \\
\hline 52 & Fosthiazate & 0.53 & 83.5 & 3.9 & 80.0 & 2.8 & 0.053 & 0.178 & 0.059 & 0.197 \\
\hline 53 & Halfenprox & 0.53 & 109.4 & 2.8 & 128.0 & 1.2 & 0.535 & 1.783 & 0.623 & 2.076 \\
\hline 54 & Heptachlor & 0.40 & 88.3 & 4.4 & 112.2 & 3.2 & 0.003 & 0.010 & 0.003 & 0.008 \\
\hline 55 & Heptachlor-epoxide & 0.27 & 76.8 & 3.0 & 95.0 & 4.3 & 0.019 & 0.064 & 0.012 & 0.041 \\
\hline 56 & Hexaflumuron & 0.67 & 107.6 & 13.1 & 130.4 & 5.7 & 0.009 & 0.030 & 0.304 & 1.013 \\
\hline 57 & Indanofan & 0.67 & 95.3 & 5.9 & 113.4 & 14.8 & 181.818 & 606.061 & 1.255 & 4.183 \\
\hline 58 & Indoxacarb & 1.07 & 101.0 & 3.9 & 116.8 & 11.5 & 1.043 & 3.477 & 1.186 & 3.954 \\
\hline 59 & Isazofos & 0.27 & 85.5 & 3.3 & 78.3 & 1.6 & 0.010 & 0.035 & 0.621 & 2.070 \\
\hline 60 & Isofenphos & 0.27 & 93.8 & 6.9 & 117.5 & 1.1 & 0.005 & 0.016 & 0.004 & 0.013 \\
\hline 61 & Lufenuron & 0.67 & 106.1 & 11.6 & 128.1 & 2.4 & 0.037 & 0.123 & 0.175 & 0.583 \\
\hline 62 & Malathion & 0.27 & 92.5 & 9.9 & 104.8 & 0.9 & 0.008 & 0.027 & 0.007 & 0.023 \\
\hline 63 & Mecarbam & 0.40 & 85.8 & 5.3 & 102.7 & 4.5 & 2.431 & 8.103 & 3.377 & 11.257 \\
\hline 64 & Metalaxyl & 2.67 & 77.7 & 3.9 & 71.1 & 0.8 & 0.028 & 0.092 & 0.152 & 0.508 \\
\hline 65 & Methyl pentachlorophenyl sulfide & 0.67 & 87.7 & 3.5 & 104.7 & 2.6 & 0.007 & 0.022 & 0.005 & 0.017 \\
\hline 66 & Metolachlor & 0.27 & 92.3 & 3.3 & 84.3 & 4.1 & 0.520 & 1.734 & 0.894 & 2.978 \\
\hline 67 & Mevinphos & 0.67 & 88.8 & 4.8 & 82.9 & 1.8 & 0.004 & 0.013 & 0.035 & 0.116 \\
\hline 68 & Myclobutanil & 0.27 & 89.8 & 2.4 & 89.0 & 2.3 & 0.015 & 0.051 & 0.647 & 2.157 \\
\hline 69 & Napropamide & 0.80 & 102.3 & 4.8 & 104.6 & 3.3 & 0.009 & 0.031 & 0.009 & 0.029 \\
\hline 70 & Nitrapyrin & 0.67 & 88.8 & 2.9 & 110.9 & 2.0 & 0.001 & 0.004 & 0.069 & 0.228 \\
\hline 71 & Norflurazon & 0.40 & 92.2 & 2.8 & 84.7 & 0.3 & 0.020 & 0.065 & 0.018 & 0.061 \\
\hline 72 & o,p-DDD & 0.13 & 99.0 & 5.4 & 107.0 & 10.0 & 0.001 & 0.005 & 0.001 & 0.004 \\
\hline 73 & $\mathrm{o}, \mathrm{p}-\mathrm{DDE}$ & 0.27 & 98.5 & 3.5 & 121.5 & 1.5 & 0.001 & 0.005 & 0.002 & 0.005 \\
\hline 74 & $\mathrm{p}, \mathrm{p}-\mathrm{DDE}$ & 0.13 & 95.5 & 3.8 & 116.5 & 1.7 & 0.003 & 0.011 & 0.006 & 0.021 \\
\hline 75 & p,p-DDT & 0.53 & 99.3 & 3.2 & 123.8 & 0.8 & 0.002 & 0.006 & 0.001 & 0.004 \\
\hline 76 & Paclobutrazol & 0.27 & 109.3 & 9.6 & 95.3 & 3.0 & 0.004 & 0.013 & 0.010 & 0.033 \\
\hline 77 & Parathion-methyl & 2.00 & 91.7 & 5.7 & 123.0 & 4.0 & 0.010 & 0.034 & 0.007 & 0.024 \\
\hline 78 & Penconazole & 0.13 & 87.5 & 4.6 & 88.0 & 2.3 & 0.006 & 0.021 & 0.015 & 0.052 \\
\hline 79 & Phenthoate & 0.40 & 88.8 & 5.5 & 112.2 & 1.4 & 0.006 & 0.019 & 0.004 & 0.014 \\
\hline 80 & Phosalone & 0.27 & 100.0 & 5.0 & 117.0 & 0.8 & 0.028 & 0.094 & 0.012 & 0.041 \\
\hline 81 & Phosmet & 0.27 & 101.8 & 3.7 & 108.5 & 1.7 & 0.986 & 3.285 & 0.924 & 3.079 \\
\hline 82 & Pirimiphos-ethyl & 0.27 & 90.0 & 0.8 & 91.3 & 3.0 & 0.012 & 0.040 & 0.014 & 0.047 \\
\hline 83 & Pirimiphos-methyl & 0.27 & 88.0 & 7.5 & 81.4 & 2.0 & 0.005 & 0.017 & 0.005 & 0.016 \\
\hline 84 & Procymidon & 0.27 & 91.3 & 3.7 & 102.8 & 1.5 & 0.912 & 3.041 & 1.123 & 3.742 \\
\hline 85 & Propanil & 0.93 & 91.5 & 4.1 & 103.6 & 1.7 & 0.013 & 0.044 & 0.013 & 0.044 \\
\hline 86 & Propisochlor & 0.80 & 80.3 & 2.2 & 98.6 & 2.9 & 0.022 & 0.072 & 1.862 & 6.208 \\
\hline 87 & Propoxur & 0.13 & 96.5 & 5.7 & 103.5 & 2.6 & 0.073 & 0.244 & 0.263 & 0.875 \\
\hline 88 & Prothiofos & 0.40 & 88.3 & 13.1 & 117.2 & 19.7 & 0.009 & 0.031 & 0.422 & 1.406 \\
\hline 89 & Pyrazophos & 0.67 & 108.6 & 4.2 & 125.6 & 0.6 & 0.006 & 0.020 & 0.136 & 0.453 \\
\hline 90 & Quintozene & 0.40 & 86.8 & 4.4 & 114.8 & 0.8 & 0.021 & 0.071 & 0.012 & 0.039 \\
\hline 91 & Simazine & 0.53 & 86.6 & 3.6 & 65.9 & 0.9 & 0.037 & 0.123 & 0.122 & 0.405 \\
\hline 92 & Tebupirimfos & 0.40 & 79.2 & 2.0 & 119.7 & 6.3 & 0.017 & 0.056 & 0.010 & 0.034 \\
\hline
\end{tabular}


Table 2. Continued

\begin{tabular}{|c|c|c|c|c|c|c|c|c|c|c|}
\hline \multirow[b]{2}{*}{ No. } & \multirow[b]{2}{*}{ Pesticide } & \multirow[b]{2}{*}{$\begin{array}{l}\text { Treatment } \\
\text { conc.(ppm) }\end{array}$} & \multicolumn{2}{|c|}{ Bean } & \multicolumn{2}{|c|}{ Lemon } & \multicolumn{2}{|c|}{ Bean } & \multicolumn{2}{|c|}{ Lemon } \\
\hline & & & $\begin{array}{c}\text { Recovery } \\
(\%)\end{array}$ & $\begin{array}{c}\text { RSD } \\
(\%)\end{array}$ & $\begin{array}{c}\text { Recovery } \\
(\%)\end{array}$ & $\begin{array}{l}\text { RSD } \\
(\%)\end{array}$ & $\mathrm{ng} / \mathrm{g}$ & $\mathrm{ng} / \mathrm{g}$ & $\mathrm{ng} / \mathrm{g}$ & $\mathrm{ng} / \mathrm{g}$ \\
\hline 93 & Tecnazene & 0.27 & 83.3 & 2.6 & 103.5 & 1.5 & 0.014 & 0.047 & 0.008 & 0.028 \\
\hline 94 & Tefluthrin & 0.67 & 89.5 & 1.1 & 112.7 & 2.3 & 0.026 & 0.085 & 0.017 & 0.056 \\
\hline 95 & Tetraconazole & 0.40 & 85.0 & 5.1 & 83.0 & 7.1 & 0.017 & 0.057 & 0.027 & 0.089 \\
\hline 96 & Tetradifon & 0.53 & 96.8 & 3.0 & 111.3 & 1.6 & 0.017 & 0.056 & 0.012 & 0.041 \\
\hline 97 & Thiazopyr & 0.40 & 113.8 & 12.3 & 72.5 & 3.5 & 116.129 & 387.097 & 150.000 & 500.000 \\
\hline 98 & Thifluzamide & 0.27 & 104.8 & 4.4 & 113.0 & 2.8 & 0.204 & 0.680 & 0.200 & 0.666 \\
\hline 99 & Thiometon & 0.53 & 80.8 & 0.8 & 98.6 & 3.6 & 0.266 & 0.888 & 7.373 & 24.578 \\
\hline 100 & Tolclofos-methyl & 0.40 & 87.5 & 4.1 & 112.5 & 1.7 & 0.006 & 0.019 & 0.168 & 0.560 \\
\hline 101 & Tolyfluanid & 1.07 & 85.8 & 4.2 & 110.7 & 3.0 & 0.008 & 0.028 & 0.006 & 0.021 \\
\hline 102 & Triadimefon & 0.40 & 87.7 & 5.1 & 58.0 & 50.2 & 0.034 & 0.113 & 0.139 & 0.464 \\
\hline 103 & Triazophos & 0.27 & 108.3 & 5.7 & 107.5 & 3.4 & 0.981 & 3.271 & 1.647 & 5.491 \\
\hline 104 & Trifluralin & 0.67 & 90.7 & 5.7 & 125.3 & 2.3 & 0.019 & 0.063 & 0.010 & 0.033 \\
\hline 105 & Vinclozolin & 2.67 & 87.5 & 2.9 & 107.1 & 2.7 & 0.012 & 0.040 & 0.010 & 0.032 \\
\hline
\end{tabular}

reliable multi-residual method to determine the levels of 105 pesticides. In this study, we used the MRM parameters previously proposed. A typical chromatogram of mixture standards is shown in Fig. 2. For the MS, sensitivity was maximized by means of optimizing the amount of target ions. The parent ion was selected for each pesticide considering its $m /$ $z$ and relative abundance in order to improve sensitivity and selectivity. To obtain the sensitive peak of each pesticide, all pesticides were sorted by their retention times and divided into 15 groups. Acquisition was conducted in 15 acquisition groups according to Fig. 3. The dwell time of all MRM ions was $0.15 \sim 0.29$ sec. In this method, the scan speed of MRM ions ranged from 0.2 to $0.82 \mathrm{sec} / \mathrm{scan}$.

\subsection{Validation of the method}

Suggested method was validated for bean and lemon in order to assess its applicability to the analysis of real samples. The identification of target pesticides was carried out by searching in the appropriate retention time windows (retention time \pm $0.1 \mathrm{~min})$. The retention time data is shown in Table 1. The limits of detection (LOD : S/N $>3$ ) and quantification (LOQ: $\mathrm{S} / \mathrm{N}>10$ ) were evaluated. In lemons, the LOD and LOQ were $0.001 \sim 150 \mathrm{ng} / \mathrm{mL}$ and $0.004 \sim 500 \mathrm{ng} / \mathrm{mL}$, respectively. In case of bean sample, the LOD was $0.001 \sim 116 \mathrm{ng} / \mathrm{mL}$ and LOQ was $0.005 \sim 388 \mathrm{ng} / \mathrm{mL}$. Three uncontaminated samples of bean and lemon were spiked with the pesticides at each fortification level. Average recovery data and relative standard deviations (RSD) are shown in Table 2 . The recovery of most pesticides were between 71.1 $126.0 \%$ except for azinphos-methyl, famoxadone, fenamidone, flufenoxuron and triadimefon in lemons and $72.5 \sim 124.5 \%$ in beans, and RSD was lower than $15 \%$ in all cases. In samples used recovery test, any pesticides were not detected. The linearity of the analytical response across the studied range is excellent, with correlation coefficients higher than 0.99 in most cases, except for certain pesticides (fenoxycarb, indoxacarb, famoxadone, propisochlor and propanil).

\subsection{Application of the method to real samples}

For three years (2005 2007), the proposed method was applied to routine analysis of approximately 2,800 agricultural products in author's laboratory (KFDA, Busan, Korea). Table 3 summarizes the results found that were above the limits permitted by the legislation of Korea. A very large number of the analyzed samples (pepper leaves, chamnamul, chard, perilla leaves, shinsuncho, red mustard, welsh onion, chwinamul, crown daisy, radish leaves, marsh mallow 
Table 3. Detection frequency of pestisides residue in agricultural peoducts

\begin{tabular}{clcc}
\hline \hline No. & Pesticide & $\begin{array}{c}\text { No. of } \\
\text { frequency } \\
\text { detected }\end{array}$ & $\begin{array}{c}\text { No. over } \\
\text { MRLs } \\
\text { Korea }\end{array}$ \\
\hline 1 & Bifenthrin & 14 & 2 \\
2 & Chlorpyrifos & 9 & 4 \\
3 & Cypermethrin & 25 & 2 \\
4 & Diazinon & 8 & 2 \\
5 & Endosulfan & 18 & 1 \\
6 & EPN & 1 & 1 \\
7 & Ethoprophos & 8 & 3 \\
8 & Flufenoxuron & 16 & 2 \\
9 & Isazofos & 1 & 1 \\
10 & Metalaxyl & 8 & 1 \\
11 & Procymidon & 63 & 3 \\
12 & Vinclozolin & 1 & 1 \\
& Total & 172 & 23 \\
\hline
\end{tabular}

and carrot) exceeded the MRLs (Maximum Residual Levels) of Korea. The pesticides most frequently found at levels over Korea's MRLs were lufenuron, kresoxim-methyl, chlorpyrifos, procymidone, ethoprophos, diazinone, cypermethrin and metalaxyl. Other agricultural products analyzed presented less positive results.

\section{Conclusions}

A simultaneous multi-residual method was developed for rapid and precise confirmation of the levels of 105 pesticides using GC/MS/MS. The results obtained showed a good analytical performance of sensitivity and selectivity of the suggested method. The use of the MRM parameters supports the analytes identified. For all pesticides, the sensitivity of this method was high enough to ensure a reliable determination at levels lower than the respective MRL.

\section{References}

1. F. J. Egea Gonzalez, A. Mena Granero, C. R. Glass, A. Garrido Frenich and J. L. Martinez Vidal, Rapid Communications in mass Spectrometry, 18, 537-543(2004).

2. S. K. Cho, A. M. Abd EI-Aty, Y. S. Park, J. H. Choi, S. Khay, C. A. Kang, B. J. Park, S. J. Kim and J. H. Shim, Biomed. Chromatogr. 21, 602-609(2007).

3. J. Boer and R. J. Law, J. Chromatogr. A., 1000, 223251(2003)

4. G. F. Pang, Y. M. Liu, C. L. Fan, J. J. Zhang, Y. Z. Cao, X. M. Li, Z. Y. Li, Y. P. Wu and T. T. Guo, Anal. Bioanal. Chem., 384, 1366-1408(2006)

5. Z. Lv, L. Gao, H. Gao, Z. Hou and B. Zhang, J. Food Sci., 74, 37-41(2009).

6. X. G. Chu, X. Z. Hu and H. Y. Yaho, J. Chromatogr. A., 1063, 201-210(2005).

7. P. Sandra, B. Tienpont and F. David, J. Chromatogr. A., 1000, 299-309(2003).

8. F. J. Arrebola, J. L. Martinez Vidal, A. Fernandez Gutierrez and M. A. Akhtar, Anal. Chim. Acta., 401, 45(1999).

9. E. Pitarch, R. Serrano, F. J. Lopez, F. Hernandez, Anal. Bioanal Chem, 376, 189-197(2003).

10. E. Jaqerdeo, J. E. Schaff, M. A. Montgomery, M. A. Lebeau, Rapid Commun. Mass Spectrum., 23, 26972705(2009).

11. D. Tsikas, F. M. Gutaki, F. M., J. Sandmann, E. Schwedhelm and J. C. Frolich, J. Chromatogr. B. Biomed. Sci. Appl., 731, 285-291(1999).

12. N. Sauret-Szczepanski, P. Mirabel, H. Wortham, Environ. Pollut. 139, 133-142(2006).

13. J. L. Martinez Vidal, M. C. Pablos-Espada, A. Garrido Frenich and F. J. Arrebola, J. Chromatogr. A., 867, 235245(2000)

14. J. L. Fernandez Moreno, A. Garrido Frenich, P. Plaza Bolanos and J. L. Martinez Vidal, J. Mass Spectrom., 43, 1235-1254(2008).

15. M. Gamon, C. Lleo and A. Ten, F. Mocholi, J. AOAC Int., 84, 1209-1216(2001) 